\title{
Heterologous Expression of Daptomycin Biosynthetic Gene Cluster Via Streptomyces Artificial Chromosome Vector System ${ }^{\mathbb{S}}$
}

\author{
Seunghee $\mathrm{Choi}^{\dagger}$, Hee-Ju Nah${ }^{\dagger}$, Sisun Choi, and Eung-Soo Kim* \\ Department of Biological Engineering, Inha University, Incheon 22212, Republic of Korea
}

\author{
Received: September 15, 2019 \\ Revised: October 29, 2019 \\ Accepted: October 31, 2019 \\ First published online: \\ November 4, 2019 \\ ${ }^{*}$ Corresponding author \\ Phone: +82-32-860-8318 \\ Fax: +82-32-872-4046 \\ E-mail: eungsoo@inha.ac.kr \\ ${ }^{\dagger}$ These authors contributed \\ equally to this work. \\ S upplementary data for thi \\ paper are available on-line only at \\ http://jmb.or.kr. \\ pISSN 1017-7825, eISSN 1738-8872 \\ Copyright(C) 2019 by \\ The Korean Society for Microbiology \\ and Biotechnology
}

The heterologous expression of the Streptomyces natural product (NP) biosynthetic gene cluster (BGC) has become an attractive strategy for the activation, titer improvement, and refactoring of valuable and cryptic NP BGCs. Previously, a Streptomyces artificial chromosomal vector system, pSBAC, was applied successfully to the precise cloning of large-sized polyketide BGCs, including immunosuppressant tautomycetin and antibiotic pikromycin, which led to stable and comparable production in several heterologous hosts. To further validate the pSBAC system as a generally applicable heterologous expression system, the daptomycin BGC of $S$. roseosporus was cloned and expressed heterologously in a model Streptomyces cell factory. A 65-kb daptomycin BGC, which belongs to a non-ribosomal polypeptide synthetase (NRPS) family, was cloned precisely into the pSBAC which resulted in $28.9 \mathrm{mg} / 1$ of daptomycin and its derivatives in S. coelicolor M511(a daptomycin non-producing heterologous host). These results suggest that a pSBAC-driven heterologous expression strategy is an ideal approach for producing low and inconsistent Streptomyces NRPS-family NPs, such as daptomycin, which are produced low and inconsistent in native host.

Keywords: Streptomyces artificial chromosome, daptomycin, biosynthetic gene cluster, heterologous expression

\section{Introduction}

The screening and development of Streptomyces natural products (NPs) as antibiotics, antifungals, antivirals, immunosuppressants, and anti-cancer drugs have played essential roles in human medicine for the past several decades [1-5]. Recent post-genomic approaches have stimulated the development of microbial genome mining to identify previously unsuspected and low-titer NP biosynthetic gene clusters (BGCs) [6-9]. The heterologous expression of Streptomyces NP BGC has become an attractive method for the titer improvement and pathway engineering of a range of potentially valuable Streptomyces NPs. Because the typical size of Streptomyces NP BGC is usually larger than $20 \mathrm{~kb}$ (sometimes over $100 \mathrm{~kb}$ ), a range of strategies, such as a transformation-associated recombination (TAR) system, integrase-mediated recombination (IR) system, and plasmid Streptomyces bacterial artificial chromosome (pSBAC) system, have been developed to isolate large- sized Streptomyces NP BGCs [10-13].

The pSBAC system is an efficient tool for Streptomyces heterologous expression with site-specific restriction site insertion, recombinant pSBAC in vivo rescue, and E. coliStreptomyces intergeneric conjugation [13]. Previously, meridamycin (MER, $\sim 95 \mathrm{~kb}$ ), tautomycetin (TMC, $\sim 80 \mathrm{~kb}$ ), and pikromycin (PIK, $\sim 60 \mathrm{~kb}$ ) BGCs were isolated successfully as a single giant recombinant plasmid using the pSBAC system and those BGCs were expressed functionally in several Streptomyces cell factories, such as S. lividans and S. coelicolor [12-14]. These results showed that the pSBAC system can be an effective platform technology for the precise cloning and functional overexpression of largesized BGC of any potentially valuable low-titer metabolite in Streptomyces and its physiologically related actinomycetes. Because all three BGCs previously cloned in the pSBAC system belong to the polyketide synthase (PKS) family, it could be argued whether a different structural family BGCs, such as non-ribosomal peptide synthase (NRPS), are 
also feasible for pSBAC-driven heterologous expression.

Daptomycin is an FDA-approved highly-valuable antibiotic exhibiting strong bioactivity against Gram-positive pathogens, such as methicillin-resistant Staphylococcus aureus (MRSA), vancomycin-resistant Staphylococcus aureus (VRSA), vancomycin-resistant enterococci (VRE), and other antibiotic resistant strains [15-17]. Daptomycin belongs to A21978C family of lipopeptides containing thirteen amino acids produced by $S$. roseosporus [18]. The three major components, A21989 $C_{1-3}$, have 11-, 12- or 13-carbon branched-chain fatty acids, respectively, attached to the terminal amino group of L-Trp [18]. After the fatty acid side chains of A21978C are removed by incubation with Actinoplanes utahensis or with the $A$. utahensis deacylase produced by recombinant $S$. lividans, daptomycin can be produced by reacylation of the cyclic peptide with n-decanoyl fatty acid [19]. Alternatively, for daptomycin production biosynthetically, decanoic acid can be supplied to cultures of $S$. roseosporus to allow the direct incorporation of the straight chain supplement [20].

Previously, the heterologous expression of daptomycin BGC was performed to improve the production and generate novel analogues. A $128-\mathrm{kb}$ fragment and a $157-\mathrm{kb}$ fragment, including $65-\mathrm{kb}$ daptomycin BGC, were isolated by the construction of a BAC library and IR system, respectively [21]. Because these clones contained huge extra sequences, which are not related to daptomycin production, it might impart some genetic burden to the heterologous host. Here, the exact daptomycin BGC was isolated precisely using the pSBAC system and the recombinant pSBAC was introduced into a model surrogate host, S. coelicolor M511 [22]. This was the first example of NRPS BGC to be cloned into the pSBAC-driven heterologous expression system. The daptomycin and its derivatives were produced successfully in the heterologous host, and their levels of production varied according to decanoic acid feeding into the culture media.

\section{Material and Method}

\section{Bacterial Strains and Culture Media}

Table 1 lists the bacterial strains and plasmids used in this study. E. coli strains were cultured in Luria-Bertani (LB) broth or agar supplemented with the appropriate antibiotics at $37^{\circ} \mathrm{C}$. Spores and hyphal fragments of S. roseosporus ATCC 31568 were grown on DA1 agar medium $(0.4 \%$ glucose, $1 \%$ malt extract, $0.4 \%$ yeast extract, $0.2 \% \mathrm{CaCO}_{3}$, and $1.5 \%$ agar) for five days at $30^{\circ} \mathrm{C}$ [23]. The MS agar medium was used for the sporulation of S. coelicolor. For the production of daptomycin and its derivatives, S. coelicolor were grown in TSB media for two days and cultured for five days in $\mathrm{R} 5$ media at $30^{\circ} \mathrm{C}$. Conjugation was carried out on a modified ISP4 medium.

Insertion of Recombinant pSBAC in Vicinity of Daptomycin Biosynthetic Gene Cluster

attP-int $_{\mathrm{DBT1}}$ was removed from pSBAC by AvrII digestion and

Table 1. Bacterial strains and plasmids used in this study.

\begin{tabular}{|c|c|c|}
\hline Strain/plasmid & Relevant characteristics & Source/reference \\
\hline \multicolumn{3}{|l|}{ Plasmid } \\
\hline pATTP & attP-int $_{\Phi \mathrm{C} 31}$-containing pGEM-T easy vector & This work \\
\hline pSBAC & accIII(IV), oriT, attP-int, backbone of pCC1BAC & [10] \\
\hline pSA & Modified pSBAC which deleted attP-int & [11] \\
\hline pSAMD & Modified pSBAC with deleted attP-int and inserted $d p t P$ fragment and NheI site & This work \\
\hline pDPT & pSAMD with 70kb DNA insert containing whole $d p t$ gene cluster & This work \\
\hline pDPT001 & pDPT with attP-int ${ }_{\Phi \subset 31}$ & This work \\
\hline \multicolumn{3}{|l|}{ E. coli } \\
\hline EPI300 & $\begin{array}{l}\text { F- } m r c-A-D(m r r-h s d \text { RMS- } m c r B C) \text { trfA host for cloning and amplification of various } \\
\text { BAC vectors and constructs derived from it }\end{array}$ & Epicenter \\
\hline S17-1 & E. coli host for transferring various plasmids into Streptomyces via conjugation & \\
\hline ET12567/pUZ8002 & E. coli host for transferring various plasmids into Streptomyces via conjugation & \\
\hline \multicolumn{3}{|l|}{ Streptomyces roseosporus } \\
\hline ATCC31568 & Original daptomycin-producing strain & [18] \\
\hline \multicolumn{3}{|l|}{ Streptomyces coelicolor } \\
\hline M511 & AactII-orf4-deleted M145, Non daptomycin-producing strain & [20] \\
\hline DPT101 & M511 with pDPT001 & This work \\
\hline
\end{tabular}


ligation (named pSA) to isolate the daptomycin biosynthetic gene cluster from the chromosome by NheI digestion and ligation. To integrate the pSA into the vicinity of $\operatorname{dptP}$ by homologous recombination, a 1,720-bp DNA fragment (HR fragment) was amplified by PCR using the genomic DNA of the S. roseosporus ATCC31568 wild-type strain as a template. The amplified PCR products were analyzed by electrophoresis in $1 \%(\mathrm{w} / \mathrm{v})$ agarose gel and purified using a DNA extraction kit. The amplified PCR products were then ligated into RBC T\&A cloning vector (Real Biotech Co., Taiwan). The ligated vector was sequenced completely to confirm its integrity (Macrogen, Korea). The HR fragment, which was digested using EcoRI and HindIII, was cloned into pSA to generate pSAMD. The pSAMD was then introduced into E. coli S17-1 and conjugated directly with S. roseosporus ATCC31568 by homologous recombination. The desired mutant was selected on apramycin-included DA1 agar medium, and its genotypes were verified by PCR.

\section{Isolation of the Entire Daptomycin Biosynthetic Gene Cluster into pSBAC}

To isolate the entire daptomycin biosynthetic gene cluster, the pSAMD containing the $S$. roseosporus strain was cultured in TSB media for 1 day at $30^{\circ} \mathrm{C}$, and the genomic DNA of the mutant was prepared using a Wizard genomic DNA purification kit (Promega, USA). The genomic DNA was digested using the restriction enzyme, NheI, purified, and concentrated by ethanol precipitation before self-ligation using T4 ligase (TaKaRa, Japan). After desalting, the ligation mixture was used for the electroporation of $E$. coli EPI300. Recombinant colonies were selected on an apramycincontaining LB medium. The plasmids were isolated using a TIANprep Mini Plasmid Kit (Tiangen, China), and screened by PCR using DptM, DptA, DptA-B-C, DptD, DptI, and DptRI check primers in daptomycin cluster to identify $\mathrm{pDPT}$.

A 1,989-bp DNA fragment including the attP-int ${ }_{\Phi C 31}$ and AvrII was amplified by PCR using the pSET152 vector as a template. The amplified PCR products were analyzed by electrophoresis in $1 \%(\mathrm{w} / \mathrm{v})$ agarose gel, purified using a DNA extraction kit, and then ligated into a pGEM-T Easy vector (named pATTP). The pATTP vector was completely sequenced to ensure integrity (Macrogen, Korea). The attP-int ${ }_{\Phi \subset 31}$ was digested by AvrII and cloned into pDPT to generate pDPT001.

\section{Production and Analysis of Daptomycin and Its Derivatives}

Spores of S. coelicolor M511 and its mutants prepared from MS medium were inoculated into $250 \mathrm{ml}$ baffled flasks containing $50 \mathrm{ml}$ TSB and shaken at $230 \mathrm{rpm}$ for two days. A $1 \mathrm{ml}$ volume of seed culture was inoculated into the flasks containing $50 \mathrm{ml}$ of R5 medium and then fermented at $30^{\circ} \mathrm{C}$ on a rotary shaker at $220 \mathrm{rpm}$ for five days. Decanoic acid and methyl oleate were used as the feeding medium. After $48 \mathrm{~h}$ of fermentation, the feeding medium (final concentration, $1 \mathrm{mM}$ ) was added to the fermentation media once after $48 \mathrm{~h}$ or two, four, and six times (every 12, 18, $36 \mathrm{~h}$ ) after $48 \mathrm{~h}$. The culture broth was mixed with an equal volume of methanol to disrupt the cells and harvested by centrifugation. The supernatant was concentrated using a vacuum concentrator and dissolved in methanol. The final extracts were analyzed by HPLC (high pressure liquid chromatography) on a reverse phase column (Agilent ZORBAX SB-C18, USA) at a flow rate of $1 \mathrm{ml} / \mathrm{min}$ with UV detection at $222 \mathrm{~nm}$. Solutions $\mathrm{A}\left(\mathrm{H}_{2} \mathrm{O}\right.$ containing $\left.0.01 \% \mathrm{TFA}\right)$ and B (acetonitrile containing $0.01 \%$ TFA) were used to isolate daptomycin and the compounds of the A21978C family.

Mass spectrometric (MS) data were obtained by LC-MS analysis on Triple TOF 5600+ (AB Sciex, USA) system using electrospray ionization in positive ion mode, with a scan range of $50 \sim 2000 \mathrm{~m} / \mathrm{z}$. The LC method was run at $40^{\circ} \mathrm{C}$ on a Phenomenex Kinetex $1.7 \mathrm{u}$ C18 (2.1 mm $\times 150 \mathrm{~mm}, 1.7 \mathrm{um}$ ). The initial composition of $95 \%$ water, $10 \%$ acetonitrile and $0.01 \%$ formic acid were maintained for $3 \mathrm{~min}$, followed by a linear gradient to $95 \%$ acetonitrile and $0.01 \%$ formic acid over $21 \mathrm{~min}$, and this composition was held $25 \mathrm{~min}$ before re-equilibration; the flow rate was $0.4 \mathrm{ml} / \mathrm{min}$. The electrospray capillary voltage was $5.5 \mathrm{kV}$ and capillary temperature was maintained at $500^{\circ} \mathrm{C}$.

\section{Antibacterial Assays against Staphylococcus aureus}

The antimicrobial activities of the daptomycin and its derivatives were assessed using the paper disc diffusion method. The bioassay was performed using Staphylococcus aureus as the indicator organism. A $1 \mathrm{ml}$ sample of overnight-cultured St. aureus was mixed with $10 \mathrm{ml}$ of a sterile solution of $1 \%$ agarose in $\mathrm{H}_{2} \mathrm{O}$. A $5 \mathrm{ml}$ volume of the agarose/growth media mixture was added to a prewarmed NA medium and solidified for $30 \mathrm{~min}$. The discs (6 $\mathrm{mm}$ diameter) were saturated with $10 \mu \mathrm{l}$ of the extracts (dissolved in methanol) and placed onto an NA medium overlaid with St. aureus. After incubation for $20 \mathrm{~h}$ at $37^{\circ} \mathrm{C}$, the diameter of the inhibition zone surrounding the discs, resulting from the diffusion of bacterial compounds, was then measured.

\section{Results and Discussion}

\section{Isolation of the Daptomycin Biosynthetic Gene Cluster Using the pSBAC System}

Unique restriction enzyme site in both border regions of NP BGC is necessary to isolate the BGC using pSBAC system. While the MER BGC was isolated using the unique restriction enzyme $M f e I$ sites present in the border region of MER BGC, artificial XbaI or HindIII sites were inserted precisely near the borders of the TMC BGC and PIK BGC to capture the BGCs. No unique restriction enzyme sites were available at the border regions of TMC BGC and PIK BGC for pSBAC cloning. Fortunately, the genome sequence analysis of S. roseosporus ATCC31568 revealed two NheI restriction enzyme sites at the both border regions of the daptomycin BGC (Fig. 1). To integrate a pSBAC vector into the vicinity of the $d p t P$, a 1,720-bp gene fragment was 


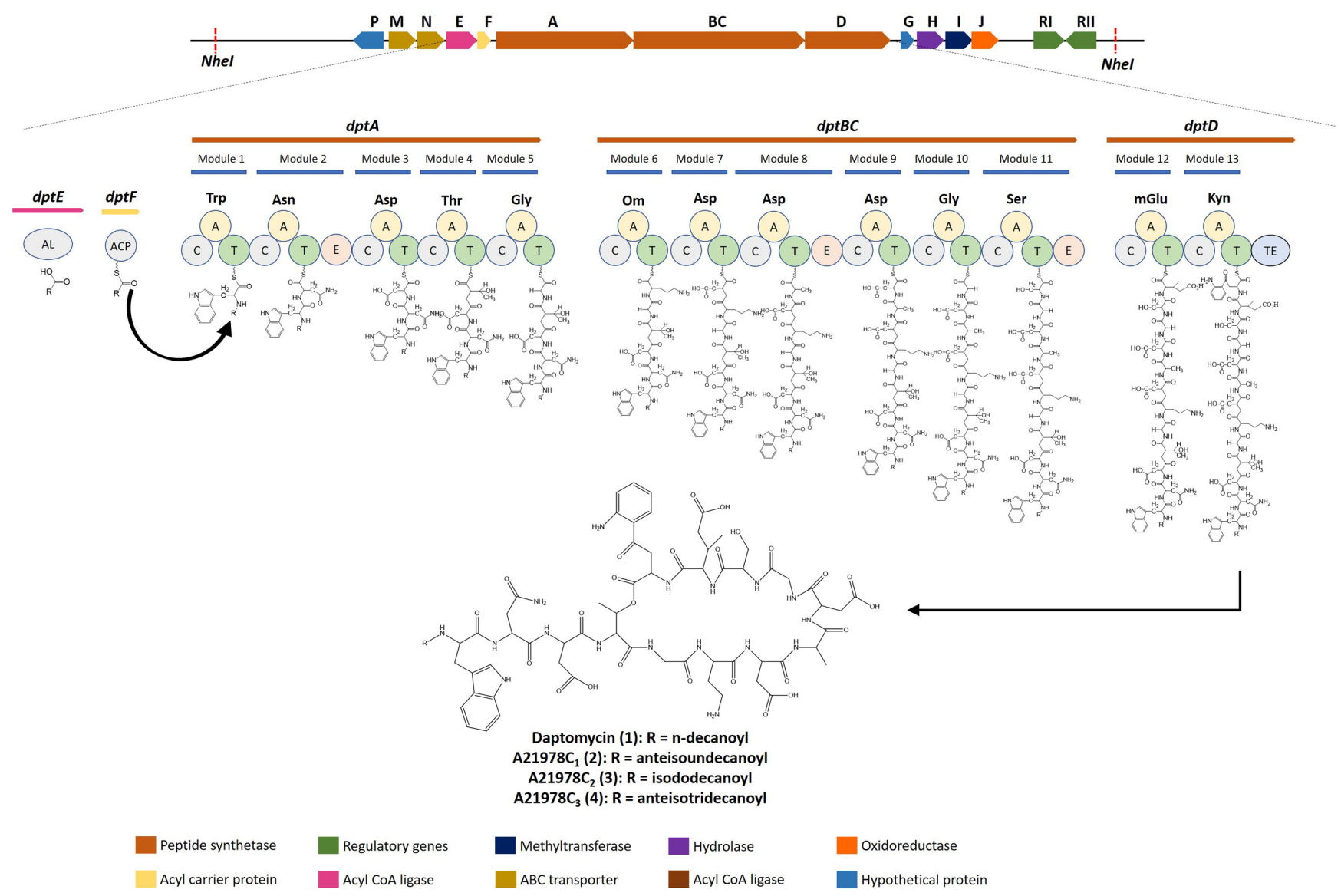

Fig. 1. Daptomycin biosynthetic pathway and its biosynthetic gene cluster.

cloned into a pSA using In-Fusion cloning kit. The resulting construct was called pSAMD, which was then introduced directly into the daptomycin-producing $S$. roseosporus. The integration of pSAMD was confirmed by PCR analysis (data not shown). The NheI-digested total chromosomal DNA fractions were then self-ligated and transformed directly into E. coli EPI300 (Fig. 2). The transformants were selected using apramycin antibiotics and confirmed by PCR (Fig. S1). As a final step of pSBAC cloning, attP-int $t_{\Phi C 31}$ was introduced into the daptomycin BGC-captured vector for the stability of daptomycin BGC in a heterologous host. The isolated TMC BGC or PIK BGC were expressed stably in Stretpomyces cell factories via integration using ФBT1 integrase. On the other hand, the integration efficiency of the ФBT1 attP-int system was quite low. Therefore, to increase the integration efficiency, the ФС31 attP-int system, which is an advantage for high integration efficiency and a broad host range, was utilized for the integration of daptomycin BGC into S. coelicolor. The AvrII-digested attP- int $_{\Phi C 31}$ from pATTP was ligated with $\mathrm{pDPT}$, and the entire daptomycin BGC-containing modified pSBAC, called pDPT001, was constructed successfully for heterologous expression.

Using the pSBAC system, the precise cloning of the target cluster can be achieved through site-specific chromosomal integration of the vector and unique restriction sites as well as in vivo plasmid rescue. Previously, the daptomycin biosynthetic gene cluster was cloned by the construction of the BAC library, but approximately the $60 \mathrm{~kb}$ region of the BAC clone was not associated with the daptomycin biosynthetic gene cluster. Here, the daptomycin biosynthetic gene cluster $(\sim 65 \mathrm{~kb})$ was isolated precisely using pSBAC and endogenous restriction enzyme, NheI, sites. Therefore, isolation of the daptomycin biosynthetic gene cluster can be another example of the isolation of natural product biosynthetic gene cluster belonging to not only type I polyketide, but also non-ribosomal polypeptide using the pSBAC system. 


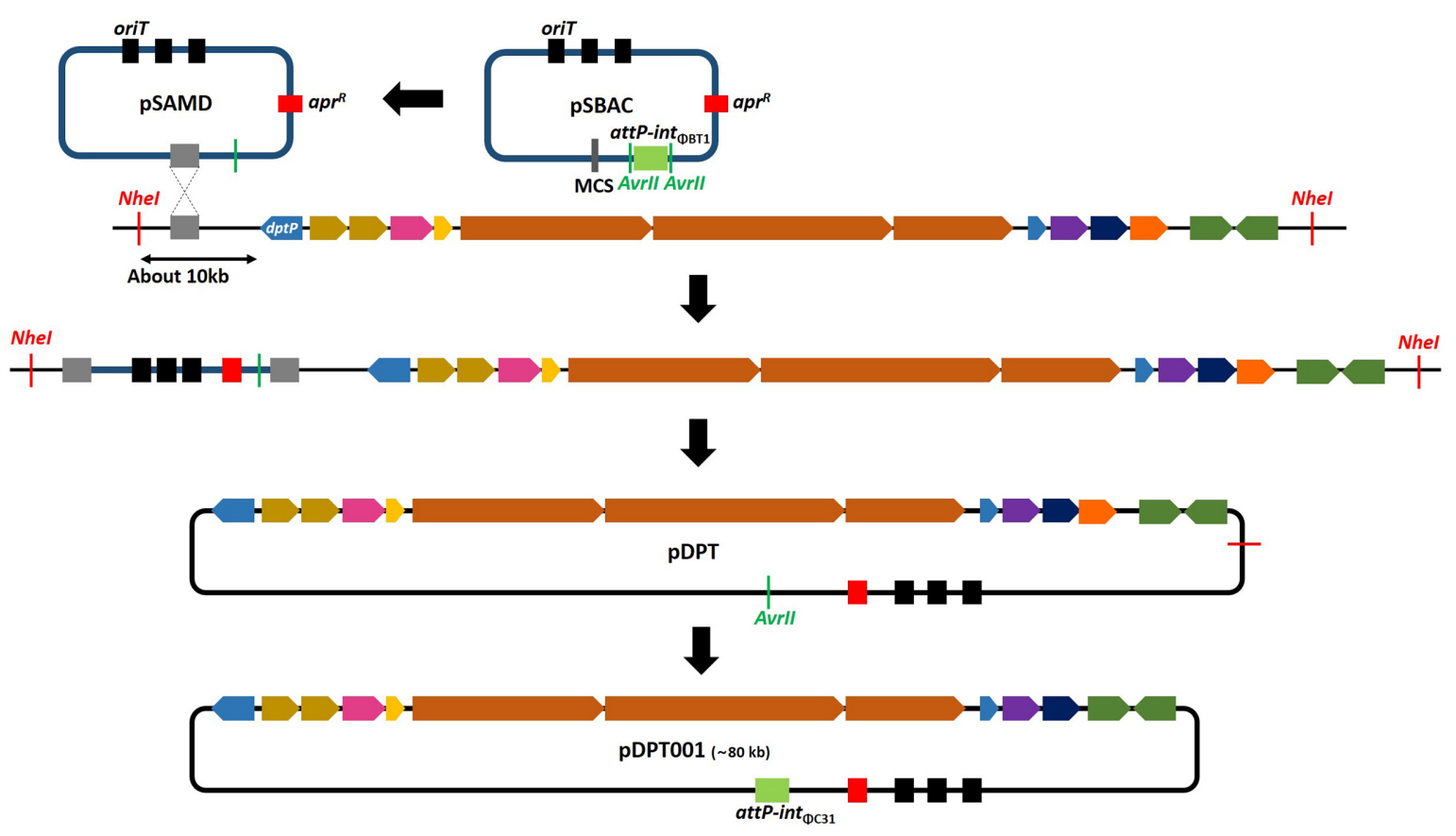

Fig. 2. Schematic description of pDPT001 construction.

Modified pSBAC called pSAMD was constructed by removing attP-int $t_{\triangle \mathrm{BT} 1}$ and inserting 1,720-bp sequences in the vicinity of $d p t P$. pSAMD was then introduced into the chromosomal DNA via homologous recombination. The daptomycin biosynthetic gene cluster was isolated by NheI digestion of the modified chromosomal DNA and its self-ligation, called pDPT. A DNA fragment containing attP-int ${ }_{\Phi C 31}$ was inserted into the AvrII recognition site of pDPT to generate pDPT001.

\section{Heterologous Expression of Daptomycin Biosynthetic} Gene Cluster in Streptomyces coelicolor

To confirm the heterologous and functional expression of the daptomycin BGC, the newly formed pDPT001 was introduced into $S$. coelicolor M511 through conjugation (named S. coelicolor DPT101). S. coelicolor M511 is one of the most widely used Streptomyces surrogate strains for heterologous expression, which is variant of $S$. coelicolor M145 with deletion of actII-orf4 for repression the actinorhodin cluster that might be a competitive pathway for heterologous BGC. The constructed strain was cultured along with the parental strain, S. coelicolor M511. HPLC analysis revealed highly noticeable peaks showing identical retention times to the authentic daptomycin only in the S. coelicolor DPT101 (Fig. 3A). LC-MS analyses with the collected fraction of the peak between $26 \mathrm{~min}$ and $28 \mathrm{~min}$ showed that the $m / z$ of $\mathbf{1}$ was 810.8630 , and its UV max was approximately $222 \mathrm{~nm}$, which is characteristic of the daptomycin. This suggests that daptomycin was produced heterologously in S. coelicolor M511 (Fig. S2). Compounds 2, 3, and 4 were also identified as daptomycin derivatives, A21798C $C_{1-3}$ (Fig. S2). A21978C $\mathrm{C}_{1-3}$ containing a cyclic anionic 13-amino acid lipopeptide can be distinguished by the 11 to 13-carbon branched-chain fatty acyl moiety attached to the $\mathrm{N}$-terminal L-Trp. This fatty acid comprises $n$-decanoic acid for daptomycin, anteiso-undecanoic acid, iso-dodecanoic acid, and anteiso-tridecanoic acid for $\mathrm{A}_{2} 1798 \mathrm{C}_{1-3}$, respectively. In contrast, the production of daptomycin and its derivatives were inconsistent in $S$. roseosporus (data not shown); the production yields of daptomycin were $3.24 \mathrm{mg} / 1$ and the total yields of daptomycin and its derivatives were $24.22 \mathrm{mg} / 1$ in the heterologous host (Fig. 3B). This shows that the pSBAC-driven heterologous expression of an entire daptomycin BGC resulted in consistent production of daptomycin in a surrogate host S. coelicolor. As expected, the antimicrobial activity against Staphylococcus aureus was detected only in the extracts of DPT101 (Fig. 3C).

Previously, the attP-int $t_{\Phi \mathrm{BT} 1}$ system was used for the stable expression of TMC BGC and PIK BGC in heterologous hosts, despite its integration efficiency being very low. In this study, the phage ФС 31 attP-int system, which has an advantage for high integration efficiency, was utilized for the integration of daptomycin BGC into S. coelicolor. After conjugation, many colonies ( $>30$ colonies) were shown in the ФС31 system while only a few colonies were observed in the ФBT1 system (data not shown). Integration into the 
A

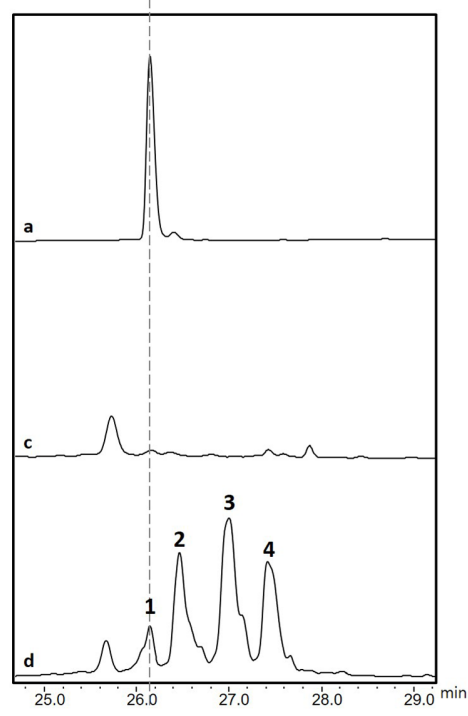

B

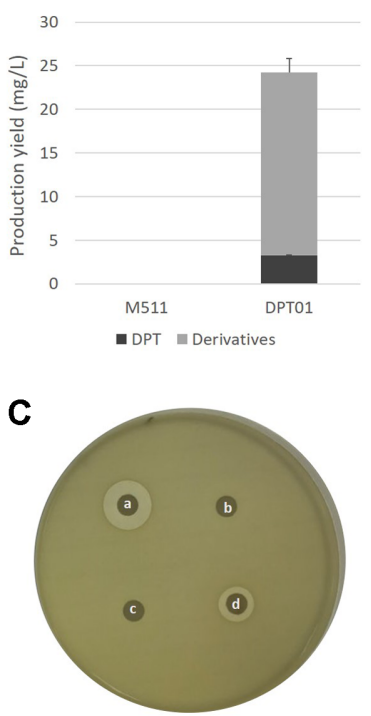

Fig. 3. (A) HPLC analysis of DPT101. (B) Production yield of daptomycin and its derivatives. (C) Antimicrobial assay against Staphylococcus aureus. a, authentic daptomycin $5 \mathrm{mg}$; b, culture media with decanoic acid; c, S. coelicolor M511 wild type; d, S. coelicolor DPT101.

$\Phi C 31$ attB site in $S$. coelicolor did not affect the production of daptomycin and its derivatives. These results suggest that the pSBAC system combined with the $\Phi C 31$ system can be used for the development of various NRPS-driven NP BGCs.

\section{Heterologous Production of Daptomycin Via Decanoic Acid Feeding}

The biosynthesis of daptomycin is initiated by the condensation of decanoic acid (a 10-carbon branched chain fatty acid) and L-Trp [24]. Therefore, the addition of decanoic acid to the fermentation medium is essential for daptomycin production [20]. However, decanoic acid feeding should be optimized because high concentration of decanoic acid could affect the surrogate host cell growth. To improve daptomycin production in a $S$. coelicolor DPT101 strain, $1 \mathrm{mM}$ decanoic acid was added to the shake flask culture once after $48 \mathrm{~h}$ or two, four and six times after $48 \mathrm{~h}[25,26]$. Compared to repeated feeding of decanoic acid, two, four and six mM of feeding medium is added among the cultures tested. Although precursor was fed repeatedly or once in higher concentration, it resulted in similar level of daptomycin and its derivatives production (Fig. 4). These results suggested that adding higher amount of decanoic acid has no effect on improvement of

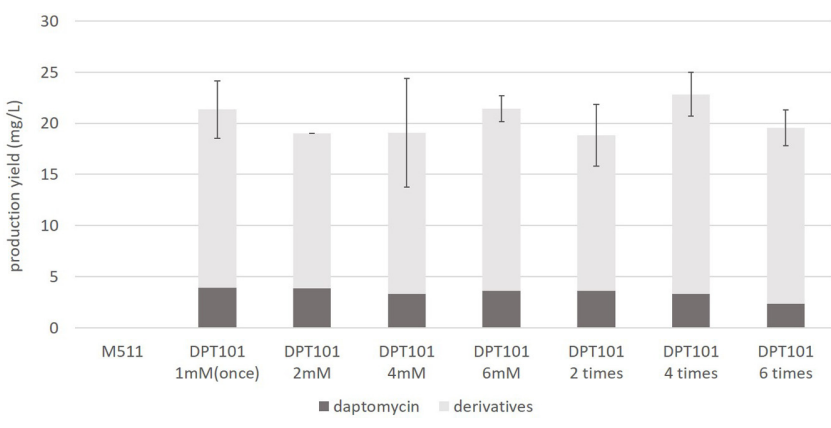

Fig. 4. Comparison of the production of daptomycin and its derivatives by optimization of the number of times decanoic acid was fed in DPT101.

Quantitative production yield of daptomycin and its derivatives.

daptomycin production yield for S. coelicolor DPT101. Increasing daptomycin production yield in a heterologous strain still needs further improvement with several strategies such as BGC refactoring, regulatory network optimization, and heterologous expression in more diverse Streptomyces cell factories. In summary, the $65-\mathrm{kb}$ daptomycin BGC was cloned successfully in a single pSBAC vector system and expressed functionally in a heterologous host, suggesting that a pSBAC-driven heterologous expression strategy is an efficient approach for the production of Streptomyces NRPS-family NP BGC.

\section{Acknowledgements}

This study was supported by "National Research Foundation of Korea (NRF) (Project No. NRF-2017R1A2A2A05069859). This work was also funded by Agricultural Microbiome R\&D Program, Ministry of Agriculture, Food and Rural Affairs, Republic of Korea (as part of the (multi-ministerial) Genome Technology to Business Translation Program). No. 918008-04.

\section{Conflict of Interest}

The authors have no financial conflicts of interest to declare.

\section{References}

1. Clardy J, Fischbach MA, Walsh CT. 2006. New antibiotics from bacterial natural products. Nat. Biotechnol. 24: 1541-1550.

2. Jang JP, Han JM, Jung HJ, Osada H, Jang J, Ahn JS. 2018. Anti-Angiogenesis effects induced by Octaminomycins A and B against HUVECs. J. Microbiol. Biotechnol. 28: 1332-1338. 
3. Lam KS. 2007. New aspects of natural products in drug discovery. Trends Microbiol. 15: 279-289.

4. Genilloud O, González I, Salazar O, Martín J, Tormo JR, Vicente F. 2011. Current approaches to exploit actinomycetes as a source of novel natural products. J. Ind. Microbiol. Biotechnol. 38: 375-389.

5. Alshaibani M, Zin NM, Jalil J, Sidik N, Ahmad SJ, Kamal N, et al. 2017. Isolation, purification, and characterization of five active diketopiperazine derivatives from endophytic Streptomyces SUK 25 with antimicrobial and cytotoxic activities. J. Microbiol. Biotechnol. 27: 1249-1256.

6. Medema $\mathrm{MH}$, Blin $\mathrm{K}$, Cimermancic $\mathrm{P}$, de Jager $\mathrm{V}$, Zakrzewski P, Fischbach MA, et al. 2011. antiSMASH: rapid identification, annotation and analysis of secondary metabolite biosynthesis gene clusters in bacterial and fungal genome sequences. Nucleic Acids Res. 39: W339-W346.

7. Ikeda H, Ishikawa J, Hanamoto A, Shinose M, Kikuchi H, Shiba T, et al. 2003. Complete genome sequence and comparative analysis of the industrial microorganism Streptomyces avermitilis. Nat. Biotechnol. 21: 526-531.

8. Bentley SD, Chater KF, Cerdeño-Tárraga AM, Challis GL, ThomsonNR, James KD, et al. 2002. Complete genome sequence of the model actinomycete Streptomyces coelicolor A3 (2). Nature 417: 141-147.

9. Winter JM, Behnken S. Hertweck C. 2011. Genomicsinspired discovery of natural products. Curr. Opin. Chem. Biol. 15: 22-31.

10. Yamanaka K, Reynolds KA, Kersten RD, Ryan KS, Gonzalez DJ, Nizet V, et al. 2014. Direct cloning and refactoring of a silent lipopeptide biosynthetic gene cluster yields the antibiotic taromycin A. Proc. Natl. Acad. Sci. USA 111: 19571962.

11. Du D, Wang L, Tian Y, Liu H, Tan H, Niu G. 2015. Genome engineering and direct cloning of antibiotic gene clusters via phage BT1 integrase-mediated site-specific recombination in Streptomyces. Sci. Rep. 5: 8740.

12. Liu H, Jiang H, Haltli B, Kulowski K, Muszynska E, Feng X, et al. 2009. Rapid cloning and heterologous expression of the meridamycin biosynthetic gene cluster using a versatile Escherichia coli- Streptomyces artificial chromosome vector, pSBAC. J. Nat. Prod. 72: 389-395.

13. Nah HJ. Woo MW, Choi SS, Kim ES. 2015. Precise cloning and tandem integration of large polyketide biosynthetic gene cluster using Streptomyces artificial chromosome system. Microb. Cell Fact. 14: 140
14. Pyeon HR, Nah HJ, Kang SH, Choi SS, Kim ES. 2017. Heterologous expression of pikromycin biosynthetic gene cluster using Streptomyces artificial chromosome system. Microb. Cell Fact. 16(1): 96.

15. Alborn WE Jr., Allen NE, Preston DA. 1991. Daptomycin disrupts membrane potential in growing Staphylococcus aureus. J. Antimicrob. Agents Chemother. 35: 2282-2287.

16. Tally FP, DeBruin MF. 2000. Development of daptomycin for gram-positive infections. J. Antimicrob. Chemother. 46: 523-526.

17. Micklefield J. 2004. Daptomycin structure and mechanism of action revealed. Chem. Biol. 11: 887-888.

18. Debono M, Barnhart $M$, Carrell CB, Hoffmann JA, Occolowitz JL, Abbott BJ, et al. 1987. A21978C, a complex of new acidic peptide antibiotics: isolation, chemistry, and mass spectral structure elucidation. J. Antibiot. 40: 761-777.

19. Boeck LD, Fukuda DS, Abbott BJ, Debono M. 1988. Deacylation of A21978C, an acidic lipopeptide antibiotic complex, by Actinoplanes utahensis. J. Antibiot. 41: 1085-1092.

20. Huber FM, Pieper RL, Tietz AJ. 1988. The formation of daptomycin by supplying decanoic acid to Streptomyces roseosporus cultures producing the antibiotic complex A21978C. J. Biotechnol. 7: 283-292.

21. Penn J, Li X, Whiting A, Latif M, Gibson T, Silva CJ, et al. 2006. Heterologous production of daptomycin in Streptomyces lividans. J. Ind. Microbiol. Biotechnol. 33: 121-128.

22. Gomez-Escribano JP, Bibb MJ. 2011. Engineering Streptomyces coelicolor for heterologous expression of secondary metabolite gene clusters. Microb. Biotechnol. 4: 207-215.

23. Kieser, T., M. J. Bibb, M. J. Buttner, K. F. Chater and D. A. Hopwood. 2000. Practical Streptomyces genetics. pp. 409. Vol. 3(4). John Innes Foundation, Norwich.

24. Lee SK, Kim HR, Jin YY, Yang DH, Suh JW. 2016. Improvement of daptomycin production via increased resistance to decanoic acid in Streptomyces roseosporus. J. Biosci. Bioeng. 122: 427-433.

25. Liao G, Liu Q, Xie J. 2013. Transcriptional analysis of the effect of exogenous decanoic acid stress on Streptomyces roseosporus. Microb. Cell Fact. 12: 19.

26. Zhang Q, Chen Q, Zhuang S, Chen Z, Wen Y, Li J. 2015. A MarR family transcriptional regulator, DptR3, activates daptomycin biosynthesis and morphological differentiation in Streptomyces roseosporus. Appl. Environ. Microbiol. 81: 37533765 . 